\title{
State Amplification and State Masking for the Binary Energy Harvesting Channel
}

\author{
Kaya Tutuncuoglu ${ }^{1}$, Omur Ozel ${ }^{2}$, Aylin Yener ${ }^{1}$, and Sennur Ulukus ${ }^{2}$ \\ ${ }^{1}$ Department of Electrical Engineering, The Pennsylvania State University, University Park, PA 16802 \\ ${ }^{2}$ Department of Electrical and Computer Engineering, University of Maryland, College Park, MD 20742
}

\begin{abstract}
In this paper, we consider a binary energy harvesting transmitter that wishes to control the amount of side information the receiver can obtain about its energy harvests. Specifically, we study state amplification and state masking, which define the maximum and minimum amount of state information conveyed to the receiver for a given message rate, respectively. For an independent and identically distributed energy harvesting process, we first find the amplification and masking regions for a transmitter without a battery and a transmitter with an infinite battery. Next, we find inner bounds for these regions for a unit-sized battery at the transmitter using two different encoding schemes, using instantaneous Shannon strategies and using a scheme based on the equivalent timing channel introduced in our previous work. We observe that the former provides better state amplification, while the latter provides better state masking.
\end{abstract}

\section{INTRODUCTION}

In the energy harvesting channel, the harvested energy is a random process that is revealed to the transmitter causally throughout the transmission. Since the transmitted symbols are constrained by the energy available at the encoder, the decoder obtains some information about the energy harvesting process in addition to the intended message. Depending on the application, it may be desirable to maximize or minimize this side information about the harvested energy, e.g., it may facilitate smart scheduling based on energy harvesting rates, but may also reveal the position or energy source of a wireless node. These scenarios result in the problems of state amplification [1], [2] and state masking [3], respectively.

The capacity of an energy harvesting channel is determined in previous work for two extreme cases for the AWGN channel. When the battery-size is unlimited, [4] shows that the capacity is equal to the capacity of the same system with an average power constraint equal to the average recharge rate. At the other extreme, when the battery size is zero, the system becomes a stochastic amplitude-constrained channel and the capacity in this case is achieved [5] by using Shannon strategies [6]. The capacity for the case of a finite-sized battery is open in general. In the special case of a noiseless binary channel and a unit-sized battery, [7] shows that the channel is equivalent to an additive geometric-noise timing channel, for which upper bounds and achievable rates using timing channel based encoding schemes are proposed. Concurrently, in [8], encoding schemes that utilize Shannon strategies are studied

This work was supported by NSF Grants CNS 09-64364/CNS 09-64632, and CCF 14-22347/CCF 14-22111. in a more general setting, and the corresponding achievable rates are found, where only the instantaneous battery states are used to determine channel input at each channel use. For the channel model in [7], [9] introduces a tighter upper bound and a better encoding scheme, outperforming i.i.d. and first order Markov Shannon strategies that utilize current battery state only. Reference [10] extends the model of [9] to a noisy channel with arbitrary battery size, and obtains upper bounds by providing battery state information to the receiver. The finite-sized battery model is also of interest in an AWGN channel. In [11], an approximate capacity for this channel model is found with a constant gap using amplitude constrained codebooks. For deterministic energy harvesting in an AWGN channel, [12] evaluates a lower bound on the capacity via the volume of the feasible input set.

The state amplification problem, i.e., sending information about system state along with the message, is first studied in [1]. This reference quantifies the side information revealed to the receiver as the mutual information between the output sequence and the state sequence. This problem is later considered in terms of the distortion in channel state estimation in [2]. The state masking counterpart, i.e., concealing channel state as much as possible while sending a message, is introduced in [3] for both causal and non-causal state information at the encoder. In an energy harvesting setting, the state amplification problem is first studied in [13] for an AWGN channel with infinite and no battery at the encoder.

In this paper, we evaluate state amplification and state masking regions, which outline the set of achievable message rates and state revealing rates for a binary energy harvesting channel. Our goal is to investigate the trade-off between the energy arrival information that the receiver can extract from the communication and the message transmission rate. We consider binary energy arrivals as the state of the channel, where each energy unit corresponds to the energy required to send a 1 through the binary channel once. We consider the no battery case and the infinite battery case, for which the channel capacities are known, and the unit battery case, for which we derive inner bounds using the achievable rates in [7], [8].

\section{Channel Model and Problem Formulation}

We consider a binary symmetric channel (BSC) with an energy harvesting transmitter, as shown in Fig. 1. The crossover probability of the binary symmetric channel is denoted by 


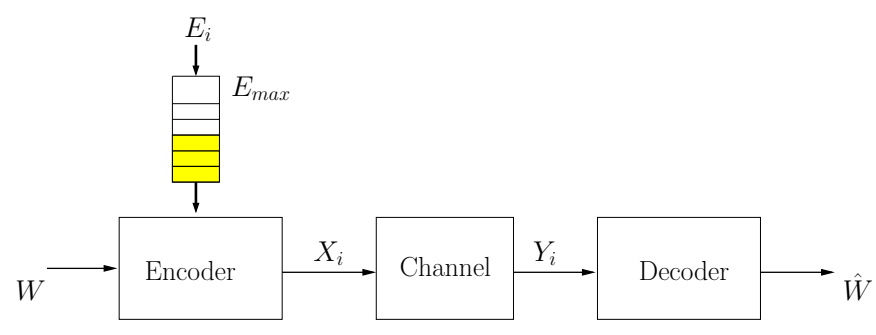

Fig. 1. The energy harvesting channel with battery size $E_{\max }$.

$p_{e} \leq \frac{1}{2}$. In the $i$ th channel use, the encoder harvests an energy of $E_{i} \in\{0,1\}$, i.e., harvests a unit of energy or not, and sends a binary symbol $X_{i} \in\{0,1\}$ through the channel. The energy cost of sending a 1 is 1 unit, while sending a zero does not require any energy. The energy arrivals $E_{i}$ are independent and identically distributed (i.i.d.) with the probability of arrival $\operatorname{Pr}[E=1]=q . E_{i}$ values are revealed to the encoder causally, while not available to the decoder. We consider two cases for the energy storage and consumption model. If the encoder has no battery, denoted by $E_{\max }=0$, then the channel input is constrained by the energy harvested within the same channel use, i.e., $X_{i} \leq E_{i}$. For $E_{\max }>0$, we consider a transmit-first model. Denoting the state of the battery at the beginning of the $i$ th channel use as $B_{i}$, the encoder first transmits $X_{i} \leq B_{i}$, and then stores the harvested energy $E_{i}$ in the battery, provided that the battery capacity permits storage. Hence, the battery state evolves as

$$
B_{i+1}=\min \left\{B_{i}-X_{i}+E_{i}, E_{\max }\right\} .
$$

In this work, we are interested in how much the decoder can learn about the energy arrival process $E^{n}$. From the decoder's perspective, there are $2^{H\left(E^{n}\right)}$ possible energy arrival sequences, since $2^{H\left(E^{n}\right)}$ is the size of the typical set for $E^{n}$. Upon receiving $Y^{n}$, the decoder can reduce the size of this list to those that are possible given $Y^{n}$, which has a size of $2^{H\left(E^{n} \mid Y^{n}\right)}$. Hence, the reduction in the entropy of $E^{n}$ for the decoder can be expressed as

$$
\Delta=\frac{1}{n}\left(H\left(E^{n}\right)-H\left(E^{n} \mid Y^{n}\right)\right)=\frac{1}{n} I\left(E^{n} ; Y^{n}\right) .
$$

Note that the value of $\Delta$ is related strongly to the encoding scheme adopted by the encoder. For example, the encoder can choose to send $X_{i}=0$ for all $i$, thus achieving no message rate, but obtaining $\Delta=0$. On the other extreme, the encoder can choose $X_{i}=E_{i}$, once again achieving no message rate, but obtaining $\Delta=H\left(E^{n}\right)$. For a non-zero rate, different encoding schemes achieving the same rate may yield different values for $\Delta$.

\section{A. State Amplification Problem}

In the state amplification problem, the encoder wishes the decoder to obtain as much information as possible about the energy harvesting process $E^{n}$, i.e., maximize $\Delta$, while reliably conveying a message with some rate $R$. This problem is first considered in [1], where the achievable message rates and state amplification rates are shown to satisfy

$$
\begin{aligned}
R & \leq I(U ; Y), \\
\Delta & \leq H(S), \\
R+\Delta & \leq I(X, S ; Y),
\end{aligned}
$$

for a memoryless channel with state $S$ known causally at the transmitter. Here, $U$ is an auxiliary random variable yielding the joint distribution $p(s) p(u) p(x \mid u, s) p(y \mid x, s)$.

\section{B. State Masking Problem}

The state masking problem, studied first in [3], finds a lower bound on $\Delta$ for any given message rate $R$. Hence, it indicates the minimum amount of information $\Delta$ that must be revealed to the decoder about the state in order to achieve some rate $R$. The achievable $(R, \Delta)$ regions are obtained by the union of the regions

$$
\begin{aligned}
& R \leq I(U ; Y), \\
& \Delta \geq I(S ; Y \mid U),
\end{aligned}
$$

for causally available state at the encoder [3]. Note that (3) and (6) are identical, and (7) provides a lower bound on $\Delta$ while (4) and (5) provide upper bounds.

In the remainder of this paper, we consider the state amplification and state masking problems individually for the cases $E_{\max }=0, E_{\max }=\infty$, and $E_{\max }=1$.

\section{No BATtery CASE: $E_{\max }=0$}

For $E_{\max }=0$, the energy available at channel use $i$ is $E_{i}$, which is i.i.d., and therefore the state of the channel is memoryless. Hence, the results of [1] extend directly to this case. Given the two states, $E_{i} \in\{0,1\}$, the two inputs $X_{i} \in\{0,1\}$, and the restriction $X_{i} \leq E_{i}$, there are two feasible mappings from $E$ to $X$. We refer to these mappings as strategies, and denote them as $U=(X, \bar{X})$, where $X$ is the channel input when $E=0$ and $\bar{X}$ is the channel input when $E=1$. The two feasible strategies are $(0,0)$ and $(0,1)$, corresponding to always transmitting a zero and attempting to send a 1 , respectively. For an encoding strategy with $\operatorname{Pr}[U=(0,1)]=p$, the exact $(R, \Delta)$ region for state amplification is obtained as

$$
\begin{aligned}
R & \leq H\left(p q * p_{e}\right)-p H\left(q * p_{e}\right)-(1-p) H\left(p_{e}\right), \\
\Delta & \leq H(q), \\
R+\Delta & \leq H\left(p q * p_{e}\right)-H\left(p_{e}\right),
\end{aligned}
$$

where $H(a)$ denotes the binary entropy function, and $p * q=$ $p(1-q)+(1-p) q$.

Next, we utilize the results of [3], and characterize the exact $(R, \Delta)$ region for state masking as (8) and

$$
\Delta \geq p H\left(q * p_{e}\right)-p H\left(p_{e}\right) .
$$

We remark that for a noiseless binary channel, i.e., $p_{e}=0$, the bounds for $\Delta$ in (8)-(10) and (11) match only at

$$
R=H(p q)-p H(q), \quad \Delta=p H(q) .
$$




\section{INFINITE BATTERy CASE: $E_{\max }=\infty$}

We next consider the infinite battery case, i.e., $E_{\max }=\infty$. With an infinite battery, [4] showed that a save-and-transmit scheme achieves the AWGN capacity with average transmit power. This scheme first saves energy for a negligible duration of the transmission, and then encodes as if constrained by an average power constraint only. The save-and-transmit scheme can be extended to the binary channel, yielding the capacity

$$
C_{B S C}= \begin{cases}H\left(q * p_{e}\right)-H\left(p_{e}\right), & q \leq \frac{1}{2}, \\ 1-H\left(p_{e}\right), & q>\frac{1}{2},\end{cases}
$$

with the channel input distribution $\operatorname{Pr}[X=1]=\min \left(q, \frac{1}{2}\right)$. Note that this is also the capacity of a BSC with an input constraint $\mathbb{E}[X] \leq q$, as is the case in [13] for the AWGN channel. With this observation, we present the state amplification region for this channel in the following lemma.

Lemma 1 The exact $(R, \Delta)$ region for the binary EH channel with an infinite-sized battery at the transmitter satisfies

$$
R+\Delta \leq C_{B S C}, \quad 0 \leq \Delta \leq H(q) .
$$

Proof: We first show the achievability of these $(R, \Delta)$ pairs. Clearly, the rate $R=C_{B S C}$ is achievable with the saveand-transmit scheme, which we assume to yield $\Delta=0$. Furthermore, by compressing the $E^{n}$ sequence and sending it as a part of the message, the encoder can trade any portion of the message rate $R$ with $\Delta$, provided that this portion does not exceed $H(q)$ for $q \leq 0.5$ and 1 for $q>0.5$. Due to the causal availability of $E_{i}$, this is performed in a block Markov fashion. For the converse, we write

$$
\begin{aligned}
I\left(X^{n} ; Y^{n}\right)=I\left(X^{n}, E^{n}, W ; Y^{n}\right) \\
\quad \geq I\left(E^{n}, W ; Y^{n}\right) \\
\quad=I\left(E^{n} ; Y^{n}\right)+H(W)-H\left(W \mid Y^{n}, E^{n}\right) \\
\quad \geq I\left(E^{n} ; Y^{n}\right)+H(W)-H(\epsilon)-\epsilon \log (n R) \\
\quad=n \Delta+n R-H(\epsilon)-\epsilon \log (n R)
\end{aligned}
$$

where $W$ is the message and $\epsilon$ is the decoding error probability. Here, (15) follows from the Markov chain $\left(W, E^{n}\right)-$ $X^{n}-Y^{n}$, (17) is due to the independence of $W$ and $E^{n}$, and (18) follows from Fano's inequality. Hence, whenever the decoding error probability $\epsilon$ goes to zero as $n \rightarrow \infty$, we have

$$
\Delta+R \leq \lim _{n \rightarrow \infty} \frac{1}{n} I\left(X^{n} ; Y^{n}\right) \leq C_{B S C},
$$

which concludes the converse.

For the masking problem, as emphasized in [13], since $(R, \Delta)=\left(C_{B S C}, 0\right)$ is achievable, perfect masking of the state $E^{n}$ is possible using the save-and-transmit scheme. Hence, we have $\Delta \geq 0$ as the masking lower bound.

\section{Unit BAtTery CASE: $E_{\max }=1$}

In preceding sections, we considered cases for which the channel capacity is known. Since this is not the case for $E_{\max }=1$, we are not able to determine the entire $(R, \Delta)$ region. In this section, we utilize two encoding schemes, proposed by [8] and [7], to find inner bounds on the $(R, \Delta)$ region for the binary noiseless channel with $p_{e}=0$. Although the channel is noiseless, this is a non-trivial model due to its memory and the state's dependence on the channel input, for which the capacity is an open problem.

\section{A. Instantaneous Shannon Strategies}

In [8], the Shannon strategies of [6], which are capacity achieving for a memoryless channel, are used to find achievable rates for the energy harvesting channel. These strategies are mappings from the current battery state $B_{i}$ to the channel input $X_{i}$. Given two battery states, $B_{i} \in\{0,1\}$, two input symbols $X_{i} \in\{0,1\}$, and the restriction $X_{i} \leq S_{i}$ in each channel use, there are two feasible mappings from $B$ to $X$. As in Section III, we denote these strategies as $U \in\{(0,0),(0,1)\}$, where the former gives $X_{i}=0$ for all $B_{i}$ and the latter gives $X_{i}=0$ for $B_{i}=0$ and $X_{i}=1$ for $B_{i}=1$. We consider a codebook consisting of strategy codewords $U^{n}$, generated i.i.d. with $\operatorname{Pr}[U=(0,1)]=p$. Upon selecting the codeword corresponding to the message, the encoder chooses $X_{i}$ based on $U_{i}$ and $B_{i}$ in the $i$ th channel use. The achieved message rate for this encoding scheme is given by

$$
R_{I I D}=\lim _{n \rightarrow \infty} \frac{1}{n} I\left(U^{n} ; Y^{n}\right) .
$$

To find the corresponding $\Delta$ for this encoding scheme, we first define the random variable

$$
\psi_{i}^{j}= \begin{cases}0, & E_{k}=0, \quad i \leq k<j, \\ 1, & \text { otherwise, }\end{cases}
$$

which is an indicator of whether a unit of energy has arrived or not between the $i$ th and $j$ th channel uses. We then define the set $\Psi\left(u^{n}\right)=\left\{\psi_{i_{1}}^{i_{2}}, \psi_{i_{2}}^{i_{3}}, \ldots\right\}$ as the collection of mutually exclusive indicators $\psi_{i_{k}}^{i_{k+1}}$, where $i_{1}=1$, and $i_{k}, k=2,3, \ldots$ are the channel indices that satisfy $u_{i_{k}}=(0,1)$. In other words, $\Psi\left(u^{n}\right)$ is the set of indicators that show whether energy is available or not for each attempt of sending a 1 given the strategy sequence $u^{n}$. We then write

$$
\begin{aligned}
H\left(E^{n} \mid Y^{n}\right)-H\left(E^{n} \mid Y^{n}, U^{n}\right) & =I\left(E^{n} ; U^{n} \mid Y^{n}\right) \\
& \leq H\left(U^{n} \mid Y^{n}\right) \\
& \leq H\left(W \mid Y^{n}\right) \\
& \leq H(\epsilon)-\epsilon \log (n R)
\end{aligned}
$$

where $\epsilon$ is the probability of decoding error. Here, (25) is due to $u^{n}$ being a function of message $w$, and (26) is due to Fano's inequality. Hence, whenever the error probability $\epsilon$ goes to zero as $n \rightarrow \infty$, we have

$$
\lim _{n \rightarrow \infty} \frac{1}{n} H\left(E^{n} \mid Y^{n}\right)=\lim _{n \rightarrow \infty} \frac{1}{n} H\left(E^{n} \mid Y^{n}, U^{n}\right) .
$$

With this observation, we write $\Delta$ as

$$
\lim _{n \rightarrow \infty} \frac{1}{n} I\left(E^{n} ; Y^{n}\right)=\lim _{n \rightarrow \infty} \frac{1}{n}\left(H\left(E^{n}\right)-H\left(E^{n} \mid Y^{n}, U^{n}\right)\right)
$$




$$
\begin{aligned}
& =\lim _{n \rightarrow \infty} \frac{1}{n}\left(H\left(E^{n}\right)-H\left(E^{n} \mid Y^{n}, U^{n}, \Psi\left(U^{n}\right)\right)\right) \\
& =\lim _{n \rightarrow \infty} \frac{1}{n}\left(H\left(E^{n}\right)-H\left(E^{n} \mid \Psi\left(U^{n}\right), U^{n}\right)\right) \\
& =\lim _{n \rightarrow \infty} \frac{1}{n} H\left(\Psi\left(U^{n}\right) \mid U^{n}\right)
\end{aligned}
$$

Here, (28) follows from (27), and (29) holds as $\Psi\left(U^{n}\right)$ can be obtained from $Y^{n}$ and $U^{n}$. Similarly, (30) follows since $Y^{n}$ can be obtained from $U^{n}$ and $\Psi\left(U^{n}\right)$. Finally, (31) holds since $H\left(\Psi\left(U^{n}\right) \mid U^{n}, E^{n}\right)=0$, and $U^{n}$ is independent of $E^{n}$.

What the series of equalities in (28)-(31) imply is that, observing $Y^{n}$, the decoder learns the indicators $\Psi\left(U^{n}\right)$ about $E^{n}$, and nothing more. Note that the intervals $\left(i_{k}, i_{k+1}\right)$ are disjoint, and therefore the elements of the set $\Psi\left(u^{n}\right)$ are independent. Since $\operatorname{Pr}\left[\psi_{i}^{j}=0\right]=(1-q)^{j-i}$ and $u_{i}$ are generated i.i.d. with probability $\operatorname{Pr}[U=(0,1)]=p$, (31) can be further simplified as

$$
\Delta=\lim _{n \rightarrow \infty} \frac{1}{n} \sum_{u^{n}}\left(p\left(u^{n}\right) \sum_{k} H\left((1-q)^{\delta_{k}}\right)\right)
$$

where $\delta_{k}=i_{k+1}-i_{k}$ denotes the number of channel uses between the $k$ th and $k+1$ st $u=(0,1)$ in $u^{n}$, and is distributed i.i.d. geometric with parameter $p$. As $n \rightarrow \infty$, due to the law of large numbers, the set $\Psi\left(u^{n}\right)$ has $n p$ elements, yielding

$$
\Delta=\lim _{n \rightarrow \infty} \frac{1}{n} I\left(E^{n} ; Y^{n}\right)=p^{2} \sum_{\delta=1}^{\infty}(1-p)^{\delta-1} H\left((1-q)^{\delta}\right) \text {. }
$$

Lemma 2 For the i.i.d. encoding scheme with Shannon strategies, the decrease in entropy of $E^{n}$ upon observing $Y^{n}$, i.e., $\Delta$, is equal to (33) for the noiseless channel.

\section{B. Timing-Based Encoding}

For the binary noiseless channel, [7] introduced an alternative scheme where encoding is performed on an equivalent timing channel. In the timing channel, the number of channel uses spent waiting for an energy are denoted by $Z_{i}$, and the number of channel uses between the energy arrival and the departure of a 1 are denoted by $V_{i}$. The decoder observes the number of channel uses between consecutive $1 \mathrm{~s}$, given by

$$
T_{i}=V_{i}+Z_{i}
$$

The timing representation is depicted in Fig. 2. Note that one use of the timing channel corresponds to $T$ uses of the binary channel. Since energy arrivals are i.i.d., $Z_{i}$ values are i.i.d. and geometric distributed.

The encoding scheme of [7] for frame length $N$ uses Shannon strategies $U \in\{0,1, \ldots, N-1\}$ to generate an i.i.d. codebook. For codeword $U^{m}$ and causally revealed $Z^{m}$, the encoder inserts

$$
V_{i}=\left(U_{i}-Z_{i} \bmod N\right)+1
$$

to the timing channel. Receiving $T_{i}$, the decoder can obtain $U_{i}=T_{i}-1 \bmod N$ without error. Hence, the rate achieved

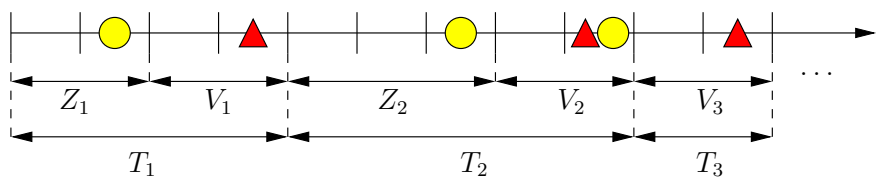

Fig. 2. Graphical representation of the variables in the equivalent timing channel $T_{n}, V_{n}$ and $Z_{n}$. Here, circles represent energy arrivals and triangles represent transmission of a 1 symbol.

with this scheme per use of the binary channel is

$$
R_{A}^{(N)}=\max _{p(u)} \frac{H(U)}{\mathbb{E}[T]} \text { bits/ch. use, }
$$

where $\mathbb{E}[T]$ is the average length of each use of the timing channel. For this encoding scheme, we next calculate $\Delta$ as a function of the strategy distribution $p(u)$. For a given output sequence $T^{m}=t^{m}$, we define $a_{i}=\sum_{j=1}^{i} t_{j}$. Then, the $i$ th use of the timing channel lies on the $a_{i}+1$ st to $a_{i}+t_{i}$ th uses of the binary channel. For this interval, the decoder can infer the following: For $t_{i} \leq N$, we have $z_{i} \leq t_{i}$ from (34). Using the definition in (22), this implies $\psi_{a_{i}}^{a_{i}+t_{i}}=1$. Otherwise, for $t_{i}>N$, we have $z_{i} \geq t_{i}-N$ since $v_{i} \leq N$ by definition. This implies that $\psi_{a_{i}}^{a_{i}+\bar{t}_{i}-N}=0$ and $\psi_{a_{i}+t_{i}-N}^{a_{i}+\overline{t_{i}}}=1$. Based on these two cases, we define the sets

$$
\begin{aligned}
& \bar{\Psi}_{0}\left(t^{m}\right)=\bigcup_{t_{i}>N} \psi_{a_{i}}^{a_{i}+t_{i}-N}, \\
& \bar{\Psi}_{1}\left(t^{m}\right)=\left(\bigcup_{t_{i} \leq N} \psi_{a_{i}}^{a_{i}+t_{i}}\right) \cup\left(\bigcup_{t_{i}>N} \psi_{a_{i}+t_{i}-N}^{a_{i}+t_{i}}\right),
\end{aligned}
$$

and write

$$
\begin{gathered}
H\left(E^{n} \mid T^{m}\right)=\sum_{t^{m}} p\left(t^{m}\right) H\left(E^{n} \mid T^{m}=t^{m}\right) \\
=\sum_{t^{m}} p\left(t^{m}\right) H\left(E^{n} \mid T^{m}=t^{m}, U^{m}=u^{m},\right. \\
\left.\bar{\Psi}_{0}\left(t^{m}\right)=0, \bar{\Psi}_{1}\left(t^{m}\right)=1\right) \\
=\sum_{t^{m}} p\left(t^{m}\right) H\left(E^{n} \mid \bar{\Psi}_{0}\left(t^{m}\right)=0, \bar{\Psi}_{1}\left(t^{m}\right)=1\right)
\end{gathered}
$$

where $\bar{\Psi}_{0}\left(t^{m}\right)=0$ denotes element-wise equality for all elements of the set $\bar{\Psi}_{0}\left(t^{m}\right)$. Here, (40) holds since $u^{m}$ can be obtained from $t^{m}$, which also reveals that $\bar{\Psi}_{0}\left(t^{m}\right)=0$ and $\bar{\Psi}_{1}\left(t^{m}\right)=1$. Note that (41) is the entropy of $E^{n}$ given that parts of $E^{n}$ are zero and parts include at least one non-zero arrival. Calculating and averaging over $t^{m}$, we get

$$
\frac{1}{n} I\left(E^{n} ; Y^{n}\right)=H(q)-\frac{1}{\mathbb{E}[T]} \mathbb{E}\left[\frac{\delta H(q)-H\left((1-q)^{\delta}\right)}{1-(1-q)^{\delta}}\right],
$$

where $\delta$ is a random variable denoting the length of $\psi$ terms in $\bar{\Psi}_{1}\left(t^{m}\right)$, and is distributed as

$$
\delta= \begin{cases}k, 1 \leq k<N, & \text { w.p. } p_{U}(k-1)\left(1-(1-q)^{k}\right), \\ N, & \text { w.p. } \sum_{u=0}^{N-1} p_{U}(u)(1-q)^{u+1} .\end{cases}
$$




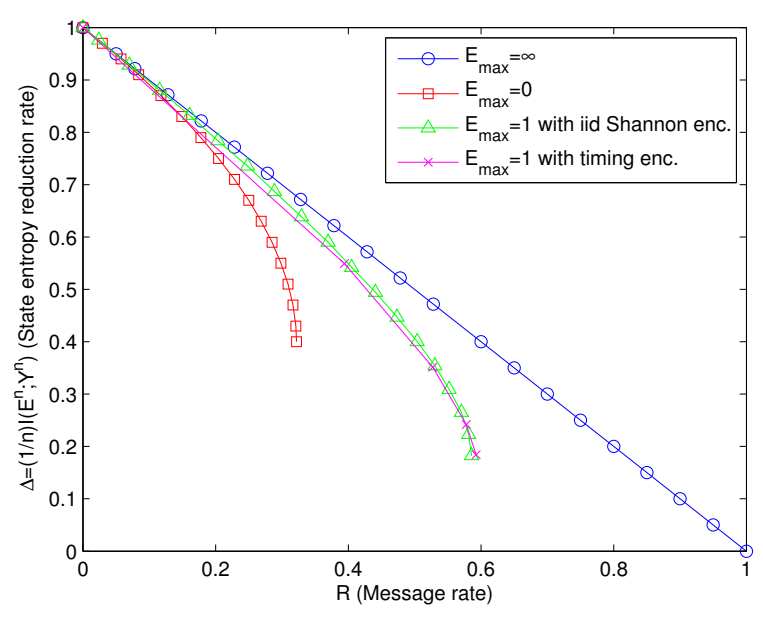

Fig. 3. The maximum $\Delta$ values with respect to message rate $R$, i.e., state amplification boundaries, for $q=0.5$ and $p_{e}=0$.

Lemma 3 For the encoding scheme in [7], the decrease in entropy of $E^{n}$ upon observing $Y^{n}$, i.e., $\Delta$, for a specific auxiliary distribution $p_{U}(u)$ is equal to (42).

Finally, we obtain $(R, \Delta)$ pairs for this encoding scheme by exhaustively searching $p_{U}(u)$ and using (36) and (42).

\section{NumERICAL RESUlts}

For comparison, we evaluate the state amplification and state masking regions for the $E_{\max }=0$ and $E_{\max }=\infty$ cases, and the maximum and minimum achievable values of $\Delta$ with respect to some message rate $R$ for the $E_{\max }=1$ case, in a noiseless channel with $q=0.5$. The state amplification results are plotted in Fig. 3, and the state masking results are plotted in Fig. 4.

In Fig. 3, we observe that the instantaneous Shannon encoding strategy in Section V-A performs state amplification almost as good as the ideal case of Section IV for low message rates. As message rate approaches the best achievable rate, state amplification is sacrificed. Moreover, we note that for the most part, instantaneous Shannon encoding provides more state information than the timing channel based encoding strategy in Section V-B.

For the state masking problem in Fig. 4, we observe that for low rates, $E_{\max }=1$ provides significantly better state masking compared to the $E_{\max }=0$ case. Similar to the state amplification case, timing-based encoding delivers less state information than instantaneous Shannon encoding, although this is desirable for the state masking case. Hence, we conclude that timing-based encoding outperforms instantaneous Shannon encoding in state masking, while the reverse is true in state amplification.

\section{CONCLUSION}

In this paper, we considered the problems of state amplification and state masking in an energy harvesting binary symmetric channel. We focused on the no battery, infinite

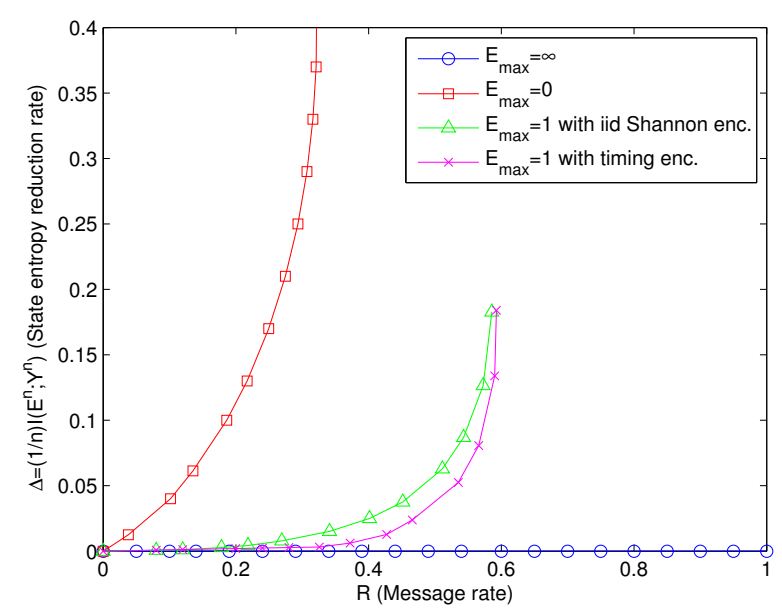

Fig. 4. The minimum $\Delta$ values with respect to message rate $R$, i.e., state masking boundaries, for $q=0.5$ and $p_{e}=0$.

battery, and unit battery cases. For the no battery case, we obtained the regions b previous results. For the infinite battery case, we found that perfect state amplification and perfect state masking are possible, in the sense that message and state information rates add up to the capacity of the channel in the case of state amplification. For the unit battery case, we compared the instantaneous Shannon strategy encoding scheme and the timing channel based encoding scheme in the noiseless case, and observed that the former provides better state amplification while the latter provides better state masking.

\section{REFERENCES}

[1] Y. H. Kim, A. Sutivong, and T. M. Cover. State amplification. IEEE Trans. on Inform. Theory, 54(5):1850-1859, May 2008.

[2] C. Choudhuri, Y. H. Kim, and U. Mitra. Causal state amplification. In IEEE ISIT, pages 2110-2114, August 2011.

[3] N. Merhav and S. Shamai. Information rates subject to state masking. IEEE Trans. on Inform. Theory, 53(6):2254-2261, June 2007.

[4] O. Ozel and S. Ulukus. Achieving AWGN capacity under stochastic energy harvesting. IEEE Trans. on Information Theory, 58(10):64716483, October 2012.

[5] O. Ozel and S. Ulukus. AWGN channel under time-varying amplitude constraints with causal information at the transmitter. In Asilomar Conference, November 2011.

[6] C. E. Shannon. Channels with side information at the transmitter. IBM Jour. of Research and Development, 2(4):289-293, 1958.

[7] K. Tutuncuoglu, O. Ozel, A. Yener, and S. Ulukus. Binary energy harvesting channel with finite energy storage. In IEEE ISIT, July 2013.

[8] W. Mao and B. Hassibi. On the capacity of a communication system with energy harvesting and a limited battery. In IEEE ISIT, July 2013.

[9] K. Tutuncuoglu, O. Ozel, A. Yener, and S. Ulukus. Improved capacity bounds for the binary energy harvesting channel. In IEEE ISIT, June 2014.

[10] O. Ozel, K. Tutuncuoglu, S. Ulukus, and A. Yener. Capacity of the discrete memoryless energy harvesting channel with side information. In IEEE ISIT, June 2014.

[11] Y. Dong and A. Özgür. Approximate capacity of energy harvesting communication with finite battery. In IEEE ISIT, June 2014.

[12] V. Jog and V. Anantharam. An energy harvesting AWGN channel with a finite battery. In IEEE ISIT, June 2014.

[13] O. Ozel and S. Ulukus. Energy state amplification in an energy harvesting communication system. In IEEE ISIT, July 2012. 